\title{
The development of a vaccination model us- ing two microorganisms and its application in leprosy and leishmaniasis
}

\author{
J CONVIT, M ULRICH, N ARANZAZU, PL CASTELLANOS, \\ ME PINARDI \& O REYES \\ Instituto de Biomedicina, Ministerio de Sanidad and Universidad Cen- \\ tral de Venezuela and Pan American Health Organization, Caracas, \\ Venezuela
}

\section{Introduction}

Conventional vaccination is oriented toward the prevention of disease in individuals capable of developing normal immune responses. A new model of vaccination employing two microorganisms has been developed in our Institute for the immunotherapy and immunoprophylaxis of two diseases, leprosy and leishmaniasis. Both of these diseases are characterized by a spectrum of clinical and histopathological manifestations.

The microorganisms causing leprosy and leishmaniasis are both intracellular pathogens in the human host, infecting principally cells of macrophage lineage; there is abundant evidence that will not be reviewed here demonstrating that effective protective immunity to both pathogens depends upon T-lymphocyte mediated reactivity. The clinical and immunological variations in human disease among different patients undoubtedly depend upon multiple factors, probably the most important of which is a variable degree of antigen-specific deficency in the development of effective cell-mediated immunity (CMI) by the host. The vaccination model which has been developed and evaluated in our studies is oriented toward correcting this antigen-specific deficiency in affected patients through the presentation of heat-inactivated specific and non-specific antigens of the infective microorganism together with viable BCG. Both immunotherapeutic and immuno-prophylactic applications of this model are discussed in this paper.

\section{Leprosy}

Control of leprosy through the conventional methods of early case detection and effective treatment of patients has not achieved the hoped-for success because of limitations which will not be reviewed here. These limitations have stimulated interest in the development of a preventive vaccine. The World Health Organization has played a fundamental role in the stimulation of these studies, particularly through the activities of the IMMLEP section of the Special Programme for Research and Training in Tropical Diseases. The association of in vivo and in vitro manifestations of CMI with more resistant forms of the disease, as well as the results of earlier studies by many investigators in patients, healthy populations and animal models have all been factors in the orientation of the model described here. The infection of nine-banded armadillos with Mycobacterium leprae, reported by Kirchheimer and Storrs in 1971 (1), provides a source of sufficient numbers of microorganisms for the practical appli- 
cation of vaccine in significant numbers of patients and healthy individuals in endemic areas. In the future, the application of the techniques of genetic engineering may provide improved sources of immunogenic products for use in this type of study.

\section{IMMUNOTHERAPY OF LEPROSY}

Antigen-specific immunodeficiency toward Mycobacterium leprae is most fully expressed in patients suffering from leprosy. Patients with lepromatous (LL) and borderline lepromatous (BL) disease show essentially no activity in either protective or hypersensitivity reactions mediated by $\mathrm{T}$ lymphocytes, though other manifestations of $\mathrm{T}$-cell activity, such as the development of suppressor cell activity and cooperation in antibody production may be intact. Patients with persistently Mitsuda-negative indeterminate leprosy (IL) and healthy contacts of leprosy patients who remain persistently Mitsuda negative probably reflect the same type(s) of antigen-specific deficiency, though to a lesser degree.

In the early 1970's we began the series of investigations which culminated in the model described here. Briefly, an in vivo test was developed which permitted the histopathological evaluation of an individual's capacity to eliminate heat-killed $M$. leprae (6.4 x $10^{7}$ acidfast bacilli) from the intracutaneous site of injection (2). Subsequently, a mixture of $6.4 \times 10^{7}$ heat-killed $M$. leprae together with $0.1 \mathrm{mg}$. BCG was injected intradermally in nonreactors to the former microorganism; histopathological evaluation of these granulomas demonstrated the development of a characteristic immune granuloma, with rapid elimination of both microorganims (3). This result was interpreted in terms of a possible macrophage defect in non-reactors to $M$. leprae, resulting in failure to present appropriate immunogens to the lymphoid system. When BCG was included, which elicits a normal response in essentially all non-reactors to $M$. leprae, we concluded the development of an immune granuloma might be accompanied by the presentation of appropriate immunogens of $M$. leprae.

Immunotherapy was initiated in a small group of patients in 1973. The results, which included remission of indeterminate lesions and the development of strong persistent Mitsuda reactivity in Mitsuda-negative contacts and inactive LL were published six years later (4). Subsequently the clinical histopathological and immunological evaluation of immunotherapy in a group of 529 weak or non-reactors to M. leprae was reported (5).

Since $1979, M$. leprae has been purified from the tissues of experimentally infected armadillos by the protocol developed by Draper (6). Each vaccine dose, administered in three intradermal sites, contains $6 \times 10^{8}$ heat-killed $M$. leprae and a variable amount $(0.01$ to 0.2 mg.) of BCG, depending upon previous reacticity to 2 IU of PPD. A complete course of treatment consists of a series of eight to ten vaccinations administered during a period of 18 to 30 months. Evaluation is based on clinical, histopathological, bacteriological and immunological criteria. Chemotherapy is continued during the course of vaccination. Prior to 1983, patients received monotherapy with sulphones; during that year, multi-drug therapy with DDS, clofazimine and rifampycin was initiated. Since the vast majority of patients in this protocol had received chemotherapy previously, their reactivity before initiating immunotherapy constitutes an internal control for several of the parameters studied subsequently.

This year an evaluation of immunotherapy in 531 patients has been completed: these results are presented in Tables 1 to 8 . 
Table 1. Classification and number of vaccinations

in leprosy patients treated with immunotherapy, 1979 to 1986.

\begin{tabular}{lcccc}
\hline Classification & $\begin{array}{c}\text { Number } \\
\text { of patients }\end{array}$ & $1-4$ & $\begin{array}{c}\text { Number of vaccinations } \\
5-7\end{array}$ & $9-10$ \\
\hline LL & & & & \\
$\quad$ Active & 227 & $17^{\mathrm{a}}$ & 29 & 181 \\
$\quad$ Inactive & 140 & 16 & 20 & 104 \\
$\quad$ Total & 367 & & & \\
BL & 77 & 7 & 14 & 56 \\
$\quad$ Active & 32 & 14 & 7 & \\
Inactive & 109 & & & \\
$\quad$ Total & & 31 & 2 & 2 \\
IL & 39 & 13 & 1 & \\
$\quad$ Active & 16 & & & \\
Inactive & 55 & & & \\
$\quad$ Total & & & & \\
\hline
\end{tabular}

a Number of patients in each group.

Table 1 shows the number of patients under control, their classification according to clinical and histopathological criteria, and the number of vaccinations which had been administered at the moment of evaluation. Although the majority of patients $(65 \%)$ had active disease, the evaluation of immunotherapy in inactive patients is also considered to be of importance in relation to possible differences in response and to induction of immunological changes which might permit controlled suspension of maintenance chemotherapy as well as reduce the risk of relapse or re-infection.

Table 2. Classification of patients receiving immunotherapy according to age.

\begin{tabular}{lrrrrrrr}
\hline \multirow{2}{*}{ Classification } & \multicolumn{8}{c}{ Age (Years) } & & \multirow{2}{*}{ Total } \\
& $<8$ & $8-12$ & $13-19$ & $20-30$ & $31-45$ & $>45$ & \\
\hline LL & 5 & 14 & 65 & 109 & 126 & 48 & 367 \\
BL & 3 & 6 & 16 & 31 & 22 & 31 & 109 \\
IL & 8 & 6 & 9 & 16 & 9 & 7 & 55 \\
\hline
\end{tabular}

Table 2 shows the distribution of the study group according to age. 
Table 3. Clinical evaluation of patients before and after immunotherapy.

\begin{tabular}{|c|c|c|c|c|}
\hline \multirow{2}{*}{ Classification } & \multicolumn{4}{|c|}{ Classification of lesions a } \\
\hline & Grade I & Grade II & Grade III & No lesions \\
\hline \multicolumn{5}{|c|}{ LL (227 patients) } \\
\hline \multirow[t]{2}{*}{ Initial } & 61 & 70 & 96 & 0 \\
\hline & $(26.8 \%)$ & $(30.8 \%)$ & $(42.2 \%)$ & $(0 \%)$ \\
\hline \multirow[t]{2}{*}{ Final } & 39 & 19 & 5 & 164 \\
\hline & $(17.1 \%)$ & $(8.3 \%)$ & $(2.2 \%)$ & $(72.2 \%)$ \\
\hline \multicolumn{5}{|c|}{ BL (77 patients) } \\
\hline \multirow[t]{2}{*}{ Initial } & 10 & 35 & 32 & 0 \\
\hline & $(12.9 \%)$ & $(45.4 \%)$ & $(41.5 \%)$ & $0 \%)$ \\
\hline \multirow[t]{2}{*}{ Final } & 18 & 5 & 0 & 54 \\
\hline & $(23.3 \%)$ & $(6.4 \%)$ & $0 \%)$ & $(70.1 \%)$ \\
\hline
\end{tabular}

a Grade I - early or minimal lesions.

Grade II - lesions of intermediate extension.

Grade III - very extensive lesions.

Table 3 presents the general clinical classification of the extension of lesions before and after immunotherapy. This Table includes all LL and BL patients with active disease at the beginning of the study; the data are not discriminated according to the number of vaccinations received. The number of both LL and BL patients with no clinically detectable lesions after immunotherapy is highly significant, as are the reductions in the numbers of patients with Grade II and Grade III lesions in both groups.

Table 4. Bacteriological indices in leprosy patients treated with immunotherapy.

\begin{tabular}{|c|c|c|c|c|c|c|c|c|}
\hline \multirow{2}{*}{ Classification } & \multicolumn{8}{|c|}{ Bacteriological index } \\
\hline & +1 & $2+$ & $3+$ & $4+$ & $5+$ & $6+$ & Negative & Not done \\
\hline \multicolumn{9}{|l|}{$\begin{array}{l}\text { Active LL } \\
\text { (227 patients) }\end{array}$} \\
\hline Initial & 31 & 27 & 31 & 22 & 26 & 82 & 0 & 8 \\
\hline Final & 23 & 19 & 9 & 2 & 0 & 0 & 151 & 23 \\
\hline \multicolumn{9}{|l|}{$\begin{array}{l}\text { Active BL } \\
\text { ( } 77 \text { patients) }\end{array}$} \\
\hline Initial & 5 & 7 & 12 & 10 & 15 & 27 & 0 & 1 \\
\hline Final & 10 & 6 & 4 & 0 & 0 & 0 & 51 & 6 \\
\hline
\end{tabular}

Bacteriological indices in patients with active LL and BL were determined before initiating immunotherapy and at the end of the experience. These results are shown in Table 4. For technical reasons, initial values are based on the bacteriological evaluation of biopsies, while the final evaluation was based on both examination of biopsies and of skin-slit smears at distant sites. The two procedures gave comparable results. The important reduction in the bacterial population in both groups of patients is clearly evident. 
Table 5. Reversal reactions evaluated histologically

in 209 biopsies from patients with active LL and BL during immunotherapy.

\begin{tabular}{cccccc}
\hline Classification & $\begin{array}{c}\text { Type of } \\
\text { reaction }\end{array}$ & $1-4$ & $\begin{array}{c}\text { Number } \\
5-7\end{array}$ & $8-10$ & Total \\
\hline LL & I & 3 & 9 & 66 & 78 \\
$(227$ patients) & II & 13 & 17 & 14 & 44 \\
& Negative & & & Subtotal LL & 136 \\
BL & I & 5 & 9 & 38 & 52 \\
( 77 patients) & II & 3 & 5 & 4 & 12 \\
& Negative & & & & 9 \\
& & & & Subtotal BL & 73 \\
\hline
\end{tabular}

a Type I- epithelioid differentiation of macrophages and lymphoid infiltration.

b Type II- lymphoid infiltration of varying degree and infiltration of small macrophages.

Reversal reactions during immunotherapy are considered to be a highly significant manifestation of cell-mediated immunological activity. This type of reactivity is invariable accompanied by an important reduction in the bacterial population of active lesions. Table 5 shows the number of reversal reactions determined by the histopathological evaluation of biopsies from 209 patients who presented suggestive clinical lesions. As might be expected, reversal reactions were more frequent in the $\mathrm{BL}$ group, where they were observed in $83.1 \%$ of the patients with active disease. Fifty-four percent of the group of patients with active LL presented histologically defined reversal reactions during the course of immunotherapy.

Table 6. Positivization of

Mitsuda reactivity in patients receiving immunotherapy; reaction size.

\begin{tabular}{lccc}
\hline Classification & Number studied & Initial Mitsuda & Final Mitsuda \\
\hline & & $\mathrm{mm} \pm \mathrm{SD}$ & $\mathrm{mm} \pm \mathrm{SD}$ \\
LL & 215 & $0.53 \pm 1.52$ & $9.79 \pm 5.39$ \\
$\mathrm{BL}$ & 79 & $1.22 \pm 2.41$ & $11.66 \pm 6.94$ \\
IL & 45 & $2.72 \pm 3.74$ & $14.80 \pm 7.97$ \\
Total & 339 & $0.99 \pm 2.28$ & $10.90 \pm 6.39$ \\
\hline
\end{tabular}

As shown in Table 6. Mitsuda reactivity increased significantly in LL, BL and IL patients after immunotherapy. This reactivity is analysed in more detail in Table 7, which relates activity to both clinical and histopathological evaluation of the Mitsuda reaction. Mitsuda reactions are applied after completing the series of eight to ten vaccinations; a significant number of patients, particularly in the LL group, have not yet been evaluated by this criterion. 
Table 7. Clinical and histological evaluation

of Mitsuda reactivity after immunotherapy with $\mathrm{M}$. leprae-BCG.

\begin{tabular}{lcccc}
\hline Classification & $\begin{array}{c}\text { Number of } \\
\text { patients }\end{array}$ & \multicolumn{2}{c}{ Mitsuda reactivity } & $\begin{array}{c}\% \\
\text { positivity }\end{array}$ \\
\hline LL & & & & \\
Clinival & 215 & 179 & 36 & $83.3 \%$ \\
reactivitya & 187 & 177 & 10 & $94.7 \%$ \\
Biopsyb & & & & \\
BL & 79 & 68 & 11 & $86.1 \%$ \\
Clinical & 59 & 58 & 1 & $98.3 \%$ \\
reactivity & & & & \\
Biopsy & 45 & 41 & $4 c$ & $91.1 \%$ \\
Clinical & & & & \\
reactivity & & & & \\
\hline
\end{tabular}

a Diameter $\geqslant 6 \mathrm{~mm}$ at 1 month.

b Presence of an epithelioid granuloma and elimination of $M$. leprae at the site of injection. (Clinically positive reactions and eight reactions of $4-5 \mathrm{~mm}$.)

c One $4 \mathrm{~mm}$ another other $5 \mathrm{~mm}$. Histological evaluation showed they were Mitsuda positive. This results in $95.6 \%$ positives.

Table 8. Classification of patients receiving immunotherapy in the clinical spectrum of leprosy, 1986.

\begin{tabular}{|c|c|c|c|c|c|c|c|c|}
\hline \multirow{2}{*}{$\begin{array}{l}\text { Initial } \\
\text { classification }\end{array}$} & \multirow{2}{*}{ Number } & \multicolumn{6}{|c|}{ Current classification } & \multirow{2}{*}{$\begin{array}{c}\text { No disease } \\
\text { activity }\end{array}$} \\
\hline & & LL & BL & BB & BT & TT & IL & \\
\hline LL & 367 & $\begin{array}{c}128 \\
(34.8 \%)\end{array}$ & $(0.8 \%)$ & $\begin{array}{c}32 \\
8.7 \%)\end{array}$ & $\begin{array}{c}6 \\
(1.6 \%)\end{array}$ & - & - & $\begin{array}{c}198 \\
(54.0 \%)\end{array}$ \\
\hline $\mathrm{BL}$ & 109 & - & $\begin{array}{c}27 \\
(24.8 \%)\end{array}$ & $\begin{array}{c}14 \\
(12.8 \%)\end{array}$ & $\begin{array}{c}6 \\
(5.5 \%)\end{array}$ & $\begin{array}{c}1 \\
(0.9 \%)\end{array}$ & - & $\begin{array}{c}61 \\
(56.0 \%)\end{array}$ \\
\hline IL & 55 & - & - & - & $\begin{array}{c}1 \\
(1.9 \%)\end{array}$ & - & $\begin{array}{c}6 \\
(11.1 \%)\end{array}$ & $\begin{array}{c}48 \\
(87.3 \%)\end{array}$ \\
\hline
\end{tabular}

Table 8 presents a global evaluation of immunotherapy in LL, BL and IL patients based on all of the criteria mentioned above, including clinical, histopathological, bacteriological and immunological parameters. Since the number of vaccinations administered was not the same in all patients, this Table reflects a dynamic situation which may show some variation in subsequent revisions. In summary, $54 \%$ of the LL patients are considered cured by the multiple criteria mentioned above: an additional $11 \%$ showed changes compatible with classification as more resistant forms of disease. Fifty-six percent of the BL group no longer show signs of disease and $19 \%$ present evidence of a shift toward increased resistance. In spi- 
te of the fact that $79 \%$ of the patients with indeterminate leprosy received four or fewer vaccinations (Table 1), $87 \%$ of the group no longer shows evidence of disease; clearly the immunological stimulus required in this type of leprosy is less than required in BL and LL.

A final commentary concerns the incidence of reactional phenomena including erythema nodosum leprosum and neuritis. When the study was begun, 189 of the original 367 patients with LL had presented phenomena of this type at some moment during the evolution of their disease. During the first six months of immunotherapy, 80 patients presented reactional phenomena of variable intensity. By the end of the third year, only eight such reactions were observed and there were none by the end of the fourth year.

The possible protective mechanisms activated by the mixture of $M$. leprae and BCG have not been fully defined, but recent evidence suggests intriguing possibilities. Lymphocytes from LL patients do not normally respond to $M$. leprae in vitro; in at least some patients, this lack of reactivity can be attributed to deficient interleukin 2 (IL-2) synthesis (7). Mehra and collaborators have reported specific antigen-induced suppressor cell activity in lepromatous leprosy; $(8,9)$ this suppressor activity is lost in some patients after vaccination $(10)$. One of the expressions of suppressor cell activity is deficient IL-2 synthesis. The normal response to BCG may provide a highly localized source of IL-2 which bypasses this defect in non-reactors to $M$. leprae. In addition, activation of macrophages by BCG may significantly alter the antigen processing and presenting functions of these cells in as yet undetermined ways, permitting the development of an effective protective response as well as cell-mediated hypersensitivity to $M$. leprae.

Immunotherapy of leprosy may be of great importance in conferring resistance to relapse or re-infection individual patients, but it would not be expected to produce a significant impact on the epidemiology of the disease. Perhaps one of the most important aspects of these studies is the demonstration that CMI to $M$. leprae can be induced in a significant number of previously unresponsive persons with the most serious and progressive forms of leprosy, and that this phenomenon is associated with protective immunity. This provides the immunological basis for the use of the same vaccination model in apparently healthy individuals at risk of being infected by $M$. leprae.

\section{IMMUNOPROHYLAXIS OF LEPROSY}

In Venezuela, initial studies of preventive vaccination in leprosy have been based on a selective approach combining epidemiological and immunological criteria to identify a potentially high-risk population of apparently healthy persons in endemic areas of leprosy. Household and non-household contacts of leprosy patients are identified through epidemiological surveys. They are skin-tested with soluble extract of $M$. leprae, a crude extract produced from purified $M$. leprae by mechanical disruption and ultrafiltration, which shows some variability among different lots, but gives an average of 60 to $75 \%$ positive reactions $(<10 \mathrm{~mm}$. at 48 $\mathrm{hr}$.) in contacts in endemic areas. Non-reactors $(<10 \mathrm{~mm}$.) are presumed to be at a relatively high risk of developing clinical disease, since they have not developed CMI to the specific or crossreacting antigens of $M$. leprae in spite of significant contact with the microorganisms and, in many cases, vaccination with BCG alone.

A preliminary trial in 2659 contacts in western Venezuela revealed $22.8 \%$ of non-reactors. Two months after vaccination with a single dose of the vaccine described above, $98 \%$ of the experimental group of previous non-reactors gave prositive reactions to $M$. leprae soluble extract; $85 \%$ were still positive after eight months. Skin test conversion is not a definitive criterion for resistance, but is one of the parameters associated with a favorable response in lepromatous patients. These encouraging results formed in part the basis for a vaccine trial supported by the World Health Organization and the Venezuelan Council for Scientific and 
Technological Research in approximately 65,000 contacts, initiated in 1984 . The preliminary observations in this much larger group do not vary significantly from the earlier results with regard to skin-test reactivity; the incidence of disease in vaccinated and control groups, which are coded in a double blind system, is still much too low for evaluation.

\section{Leishmaniasis}

American cutaneous leishmaniasis is caused by several species of flagellated protozoa belonging to the genus Leishmania, often divided into two major complexes, L. mexicana and L. braziliensis, for purposes of classification. Leishmaniasis and leprosy present many similar features, as described in the Introduction to this paper. Several significant differences might also be pointed out. In leprosy, the proportion of patients who are classified as nonreactors is quite high, particularly in some parts of the world; in Venezuela, about $50 \%$ of new patients belong to the BL-LL extreme of the spectrum. In contrast, diffuse cutaneous leishmaniasis, characterized by antigen-specific absence of protective and hyper-sensitivity manifestations of CMI to Leishmania, is a rare form of the disease. There is no good evidence for the importance of strain differences in the pathogenicity of $M$. leprae; in leishmaniasis there appears to be quite a clear association of diffuse cutaneous forms of the disease (defective CMI) with parasites belonging to the mexicana complex and muco-cutaneous forms of disease (exaggerated delayed-type hypersensitivity; incomplete protective immunity) with L. braziliensis. Nevertheless, host-dependent variations in CMI undoubtedly play a highly significant role in the immunopathology of the disease.

\section{IMMUNOTHERAPY OF LOCALIZED CUTANEOUS LEISHMANIASIS}

The favorable results obtained with immunotherapy in leprosy and preliminary results showing a favorable clinical response to the injection of heat-killed promastigotes of Leishmania together with BCG in a limited number of cases of diffuse cutaneous leishmaniasis and intermediate forms of the disease have formed the basis for a trial comparing immunotherapy and chemotherapy with Glucantime meglumine antimonate) in the treatment of localized cutaneous leishmaniasis (LCL).

Ninety-four patients with LCL were selected for study from a endemic area in northeastern Venezuela, after complete clinical and dermatological evaluation, including ECG, leishmanin skin tests and culture of lesions for secondary bacterial infection. All patients were at least 13 years old and had lesions of less than one year of evolution. Diagnosis was confirmed by histological study as well as culture in vitro and by inoculation of biopsy material into the footpads of hamsters. These patients were divided by ramdom procedures into two comparable groups; 52 received immunotherapy and 42 received Glucantime. Patients receiving immunotherapy were injected in two intradermal sites with a mixture of $6.4 \times 10^{8}$ washed, heat-killed promastigotes of L. mexicana sp. originally isolated from a patient with diffuse cutaneous leishmaniasis and 0.01 to $0.2 \mathrm{mg}$. BCG, depending upon previous reactivity to 2 IU of PPD. A second dose was applied after six to eight weeks and a third dose after an additional 12-18 weeks when necessary. Patients receiving chemotherapy were given two or three series of 20 intramuscular injections of Glucantime, with an interval of 15 days between each series. Patients in both groups were instructed in local topical treatment of their lesions with antiseptics, and antibiotics were used to treat secondary infections that persisted for more than 15 days after local treatment.

Patients were evaluated at two-week intervals during the period of treatment. Cure was determined by clinical criteria, including complete healing of ulcerated lesions, absence of inflammation or infiltration and no evidence of suspicious lesions of lymph nodes, mucous tissues or skin. Side-effects were carefully evaluated. All patients were re-examined at three- 
month intervals after clinical cure had been established; follow-up at present varies from three to 12 months. In addition to the leishmanin reaction, patients were evaluated before entry into the trial and after clinical cure by immynological tests including lymphocyte transformation tests with mitogens and promastigote antigens, assays for suppressor cells, lymphocyte subpopulations, and circulating antibodies; these results will be published in detail elsewhere. Leishmania isolates were identified by monoclonal antibodies kindly supplied by Dr. Diana McMahon-Pratt and by absorbed polyclonal sera; all isolates identified until now belong to the L. braziliensis complex.

Table 9. Clinical cure of patients treated with immunotherapy and with Glucantime.

\begin{tabular}{|c|c|c|c|c|c|c|}
\hline \multirow{3}{*}{$\begin{array}{l}\text { Weeks of } \\
\text { treatment }\end{array}$} & \multicolumn{3}{|c|}{ Immunotherapy } & \multicolumn{3}{|c|}{ Glucantime } \\
\hline & \multirow[t]{2}{*}{ Cured } & \multicolumn{2}{|c|}{ Cumulative cures } & \multirow[t]{2}{*}{ Cured } & \multicolumn{2}{|c|}{ Cumulative cures } \\
\hline & & No. & $\%$ & & No. & $\%$ \\
\hline $0-7$ & 0 & 0 & & 0 & 0 & \\
\hline $8-9$ & 1 & 1 & 1.9 & 1 & 1 & 2.4 \\
\hline $10-11$ & 4 & 5 & 9.6 & 4 & 5 & 11.9 \\
\hline $12-13$ & 8 & 13 & 25.0 & 7 & 12 & 28.6 \\
\hline $14-15$ & 13 & 26 & 50.0 & 8 & 20 & 47.6 \\
\hline $16-17$ & 10 & 36 & 69.2 & 7 & 27 & 64.3 \\
\hline $18-19$ & 0 & 36 & 69.2 & 6 & 33 & 78.6 \\
\hline $20-21$ & 0 & 36 & 69.2 & 3 & 36 & 85.7 \\
\hline $22-23$ & 0 & 36 & 69.2 & 0 & 36 & 85.7 \\
\hline $24-25$ & 1 & 37 & 71.2 & 2 & 38 & $90.5^{\mathrm{a}}$ \\
\hline $26-27$ & 3 & 40 & 76.9 & 1 & 39 & $92.9^{a}$ \\
\hline $28-29$ & 5 & 45 & 86.5 & 0 & 39 & 92.9 \\
\hline $30-31$ & 2 & 47 & 90.4 & 1 & 40 & 95.2 \\
\hline $32-33$ & 2 & 49 & 94.2 & 0 & 40 & 95.2 \\
\hline Treatment & 3 & 3 & 5.8 & 2 & 2 & 4.8 \\
\hline \multicolumn{7}{|l|}{ Failure } \\
\hline Total & 52 & 52 & & 42 & 42 & \\
\hline
\end{tabular}

a Statistical differences between the two groups $\mathrm{P}<0.05$; none of the differences at any other two-week interval was statistically significant.

The results of this comparative trial are shown in Table 9, which shows the number of patients cured at each two-week interval and the cumulative number of clinical cures as defined previously. Significant differences between the two groups were observed only during the period from 24 to 27 weeks. Since there appeared to be an arrest in cures in about $30 \%$ of the immunotherapy group after about 17 weeks of obervation, a third vaccination was given. By 33 weeks, about $95 \%$ of the patients in both groups were clinically cured. Healing occurred in an average of 16.1 week after chemotherapy and 18.3 weeks after immunotherapy; this difference is not statistically significant.

Side effects were defined as follows: Chemotherapy group - Slight: osteomuscular sensitivity, fever, headache and other manifestations that did not require suspension of treatment; moderate: similar manifestations of an intensity that required temporary suspension of treatment; severe side effects included cardio-vascular alterations or any other manifestations 
which required the definitive suspension of treatment. In the immunotherapy group, side-effects were evaluated primarily on the basis of local reactions at the vaccine injection sites.

Slight side-effects were observed in three patients $(5.77 \%$ of the total) receiving immunotherapy; no moderate or severe side-effects were observed in these patients with localized cutaneous leishmaniasis. In marked contrast, $52.38 \%$ of the patients in the group receiving chemotherapy showed side-effects, of which 19.05 and $21.43 \%$, respectively, were moderate or severe.

More than $95 \%$ of the patients had secondary bacterial infections when initially examined, caused principally by Gram-positive cocci. Only $14.9 \%$ did not respond favorably to local treatment with antiseptics and required antibiotic therapy.

Average reactions to the leishmanin test were $21.9 \pm 9.1 \mathrm{~mm}$. before treatment in the immunotherapy group and $20.5 \pm 8.1 \mathrm{~mm}$. in the chemotherapy group; after clinical cure these averages had increased to $26.8 \pm 8.7 \mathrm{~mm}$. and $24.7 \pm 8.7 \mathrm{~mm}$., respectively. The differences between the two groups are not statistically significant, but the increase after clinical cure is.

Lymphocyte transformation tests in both groups, which were initially positive did not show significant increases after clinical cure.

This study demonstrates that very similar results were obtained in the clinical response using conventional chemotherapy with Glucantime and using immunotherapy with a mixture of heat-killed promastigotes and viable BCG in the treatment of localized cutaneous leishmaniasis. The number and severity of side effects were almost negligible in the group receiving immunotherapy as compared to the group receiving antimonial chemotherapy. These observations suggest that immunotherapy offers an extremely attractive alternative in the treatment of localized leishmaniasis, in terms of applicability in primary health care centers and rural areas at highly reduced cost, with low risk of side-effects and a less rigorous requirement for specialized medical attention.

Long-term evaluation will be required to determine the efficacy of immunotherapy in preventing such complications as secondary muco-cutaneous involvement. In this trial, a strain of L. mexicana was highly effective in the therapy of disease caused by $L$. braziliensis. The incorporation of $B C G$ as an immunopotentiator may overcome some of the reported limitations in cross protection between different species of Leishmania and in the induction of immunity with non-viable microorganisms in experimental models. Studies are planned in an in vitro model which may clarify some of these questions.

\section{IMMUNOPROHYLAXIS OF CUTANEOUS LEISHMANIASIS}

Studies in leprosy clearly suggest that the model of vaccination with two microorganisms used in immunotherapy produces immunological sensitization which may be associated with protection in apparently healthy individuals, and trials are underway to evaluate this procedure. Similarly, a double-blind study of immunoprophylaxis in cutaneous leishmaniasis has been initiated in 4000 school children in an endemic area, employing a single dose of vaccine. The first observations, based on clinical and immunological criteria, will be made six months after vaccination and are not yet available for analysis.

In summary, a vaccination model employing a heat-inactivated form of the infective pathogen together with viable $\mathrm{BCG}$, has been employed in the immunotherapy of leprosy and of cutaneous leishmaniasis. Both diseases are characterized by intracellular infection in the human host and by variable degrees of antigen-specific immunodeficiency in the development of protective and hypersensitivity manifestations of cell-mediated immunity. The encouraging results observed in immunotherapy have provided the basis for trials using the same vaccination model in apparently healthy individuals in endemic areas for these two 
diseases. The applications of this model may be significantly broader than the two examples analysed in this paper. The model may be of particular interest with regard to antigenically complex microorganisms, in which multiple antigens may be involved in the induction of an effective protective response.

\section{References}

1 Kirchheimer WF, Storrs EE Attempts to establish the armadillo (Dasypus novemcinctus Linn.) as a model for the study of leprosy. 1. Report of lepromatoid leprosy in the experimentally infected armadillo. Int J Lepr, 1971; 39: 692-701.

2 Convit J, Avila JL, Goihman M, Pinardi ME. A test for the determination of competency in clearing bacilli in leprosy patients. Bull Wld Hlth Org, 1972; 46: 261-265.

3 Convit J, Finardi ME, Rodriguez Ochoa G, Ulrich M, Avila JL, Goihman-Yahr M. Elimination of Mycobacterium leprae subsequent to local in vivo activation of macrophages in lepromatous leprosy by other mycobacteria. Clin Exp Immunol, 1974; 17: 821-826.

4 Convit J, Aranzazu N, Pinardi ME, Ulrich M. Immunological changes observed in indeterminate and lepromatous leprosy patients and Mitsuda negative contacts after the inoculation of a mixture of Mycobacterium leprae and BCG. Clin Exp Immunol, 1979; 36: 214-220.

5 Convit J, Aranzazu N, Ulrich M, Pinardi ME, Reyes O and Alvarado J. Immunotherapy with a mixture of Mycobacterium leprae and BCG in different forms of leprosy and in Mitsuda-negatvie contacts. Int J Lepr, 1982; 50: 415-424.

6 Draper P. In: Problems related to purification of $M$. leprae from armadillo tissues and standardization of M. leprae preparations. Report IMMLEP, Annex 1, Feb 1979.

7 Haregewoin A, Godal T, Mustafa AS, Belehu A, Yemaneberhan T. T-cell conditioned media reverse T-cell unresponsiveness in lepromatous leprosy. Nature, 1982; 303: 342-344.

8 Mehra V, Mason LH, Fields JP, Bloom BR. Lepromin-induced suppressor cells in patients with leprosy. J. Immunol, 1979; 123: 1813-1817.

9 Mehra V, Brennan PJ, Rada E, Convit J, Bloom BR. Lymphocyte suppression in leprosy induced by unique $M$. leprae glycolipid. Nature, 1984; 308: 487-499.

10 Mehra V, Convit J, Rubinstein A, Bloom BR. Activated suppressor cells in leprosy. J Immunol, 1982; 129: 1946-1951. 